\title{
Estruturação estratégica do campo científico da Competência em Informação no Brasil: integrando redes e instituições
}

\author{
Elmira Luzia Melo Soares Simeão \\ Universidade de Brasília, Faculdade de Ciência da Informação, Brasília, DF, Brasil \\ elmira@unb.br
}

Regina Celia Baptista Belluzzo

Universidade Estadual Paulista, Programa de Pós-graduação em Ciência da Informação,

Marília, SP, Brasil

rbelluzzo@gmail.com

Fabiene Castelo Branco Diógenes*

fabiene@ibict.br

Eny Marcelino Nunes*

eny@ibict.br

Cecília Leite*

cecilia@ibict.br

*Instituto Brasileiro de Informação em Ciência e Tecnologia, Brasília, DF, Brasil

DOI: https://doi.org/10.26512/rici.v12.n2.2019.21769

Recebido/Recibido/Received: 2018-12-15

Aceitado/Aceptado/Accepted: 2019-01-08

Resumo: Busca-se ressaltar os principais estudos e vivências envolvendo o campo científico que diz respeito ao cenário atual da Competência em Informação: evidência e importância dos estudos e pesquisas no campo da Ciência da Informação no Brasil e no mundo, o papel das parcerias nacionais e internacionais, suas tendências no desenvolvimento da ciência e ações conjuntas das redes de instituições, tais como: o Instituto Brasileiro de Informação em Ciência e Tecnologia, Universidade Estadual Paulista, Universidade de Brasília e a Associação Nacional de Pesquisa e Pós-graduação em Ciência da Informação, essa última por ser a entidade mais importante para os pesquisadores dessa área no Brasil conjugando esforços de integração com outras áreas do conhecimento. Para ilustrar a relevância e atualidade do tema são apresentadas as experiências vivenciadas com essas parcerias, uma vez que lidam com práticas e lições aprendidas com o acesso e uso da informação sob a ótica da Competência em Informação, evidenciando a construção de conhecimento e sua aplicação com intervenção na realidade social, destacando-se a forma interativa em que atuam em qualquer dimensão do ensino e aprendizagem.

Palavras-chave: competência em informação. parcerias público-privadas. redes de informação. 


\section{Estructura estratégica del campo científico de la Alfabetización Informacional (ALFIN) en Brasil: integrando redes e instituciones.}

Resumen: Se busca resaltar los principales estudios y vivencias involucrando el campo científico que se refiere al escenario actual de la alfabetización informacional: evidencia e importancia de los estudios e investigaciones en el campo de la Ciencia de la Información en Brasil y en el mundo, el papel de las alianzas nacionales e internacionales, en el desarrollo de la ciencia y acciones conjuntas de las redes de instituciones, tales como: el Instituto Brasileño de Información en Ciencia y Tecnología, Universidad Estadual Paulista, Universidad de Brasilia y la Asociación Nacional de Investigación y Posgrado en Ciencia de la Información. Para ilustrar la relevancia y actualidad del tema se presentan las experiencias vivenciadas con esas alianzas, ya que se ocupan de prácticas y lecciones aprendidas con el acceso y uso de la información bajo la óptica del ALFIN, evidenciando la construcción de conocimiento y su aplicación con intervención en la intervención la realidad social, destacándose la forma interactiva en que actúan en cualquier dimensión de la enseñanza y el aprendizaje.

Palabras-clave: alfabetización informacional. alianzas públicas y privadas. redes de información.

\section{Strategic structuring of the scientific field of Information Literacy in Brazil: integrating networks and institutions.}

Abstract: It seeks to highlight the main studies and experiences involving the scientific field that refers to the current scenario of Information Literacy (IL): evidence and importance of studies and research in the field of Information Science in Brazil and the world, the role of national and international partnerships, their trends in the development of science and joint actions of the networks of institutions, such as the Brazilian Institute of Information in Science and Technology, State University of São Paulo Júlio de Mesquita Filho, University of Brasilia and the National Association of Research and Post-graduation in Information Science the latter being the most important entity for researchers in this area in Brazil, combining integration efforts with other areas of knowledge. In order illustrate your relevance and the relevance of the theme, the experiences with these partnerships are presented, since they deal with practices and lessons learned with the access and use of information from Information Literacy perspective, evidencing the construction of knowledge and its application with intervention in the social reality, highlighting the interactive form in which they act in any dimension of teaching and learning.

Keywords: information literacy. public and private partnerships. information networks.

\section{Introdução}

Recentemente, vários estudos mostram que o campo disciplinar para a "Competência em Informação"(Colnfo), tradução adotada no Brasil para o termo em inglês "Information literacy"(IL) que surgiu na década 1970, tem sido cada vez mais necessária para que os indivíduos se tornem aptos a identificar, selecionar, avaliar e usar a informação. Em tempos de uma sociedade propensa à conflitos causados por diferenças, é importante agir de forma reflexiva e ética em um processo de aprendizagem contínua e inclusiva.

Esse campo disciplinar vem se consolidando em países desenvolvidos, entretanto, o mesmo não acontece em países em desenvolvimento onde o cenário com que se depara é de grandes restrições econômicas e culturais, aspectos que influenciam fortemente o desenvolvimento e constituem barreiras à sua consolidação, por ordens práticas e estruturais principalmente. 
Em decorrência disso, algumas instituições brasileiras com foco na Ciência da Informação, pensaram em agir estrategicamente em um esforço conjunto e contínuo de cooperação, integração e de confiança. Muitas se uniram para desenvolver atividades em parcerias alicerçadas no trabalho em rede, visando a estruturação do campo científico da Colnfo na Ciência da Informação.

Em um contexto de nítido avanço tecnológico, este artigo tem como premissa ressaltar os estudos e vivências envolvendo o campo científico sobre o qual será apresentado o cenário atual da Coinfo: evidência e importância dos estudos e pesquisas no campo da Ciência da Informação no Brasil e no mundo, o papel das parcerias nacionais e internacionais, suas tendências no desenvolvimento da ciência e ações conjuntas das redes de instituições, tais como: o Instituto Brasileiro de Informação em Ciência e Tecnologia (IBICT), Universidade Estadual Paulista (Unesp), Universidade de Brasília (UnB) e a Associação Nacional de Pesquisa e Pós-graduação em Ciência da Informação (Ancib), essa última é a entidade mais importante para os pesquisadores dessa área no Brasil conjugando esforços de integração com outras áreas do conhecimento.

Para ilustrar a relevância e atualidade do tema, serão suscitadas as experiências vivenciadas com essas parcerias que lidam com práticas e lições aprendidas com o acesso e uso da informação (observando a Colnfo) para a construção de conhecimento e sua aplicação com intervenção na realidade social, e que trabalham de forma interativa em qualquer dimensão do ensino e aprendizagem.

\section{Cenário atual da COINFO}

A história desse campo temático cuja expressão Information Literacy (IL) foi criada por Zurkowski (1974) que, conforme Dudziak (2010), contou com um núcleo fundador das pesquisas da área, envolvendo bibliotecários e educadores tais como Burchinal (1976), Hamelink (1976), Taylor (1979), Breivik (1985), Kuhlthau (1987), e a Americam Library Association (ALA, 1989).

No Brasil, os estudos sobre o tema foram iniciados vinte anos depois que surgiram as primeiras pesquisas no exterior. Conforme Belluzzo (2018), a Colnfo, no Brasil, apresenta os primeiros estudos a partir da década de 2000 com os trabalhos de Caregnato (2000); Belluzzo (2001); Dudziak (2001); Campello (2002) e Hatsbach (2002), encontrando-se a área no Brasil "em estado inicial de difusão e investigação, ainda que possa se observar um crescimento significativo".

Estudos internacionais mais recentes sobre evolução da temática, cuja área de conhecimento com maior representatividade nas pesquisas, publicações e eventos é a da 
Ciência da Informação, mostram o estágio de desenvolvimento expressivo e em fase de maturação.

Como se pode observar, quando Dudziak (2010) estudou as tendências e rumos da pesquisa no campo Information Literacy (IL) e analisou a produtividade científica do tema da Competência em Informação em bases de dados internacionais e multidisciplinares da WoS (Web of Science) e Scopus, os resultados mostraram "aceitação emergente", embora com a existência de vários obstáculos na sua compreensão e a necessidade urgente da promoção da pesquisa em competência em informação em diferentes contextos e com distintos conteúdos.

Passados alguns anos se descortina um cenário mais promissor. A pesquisa de Majid et al. (2013) que explorou as tendências no campo da information literacy estudando um total de 1989 de registros do banco de dados bibliográficos Scopus, publicado 2003 a 2012 constatou que o número de publicações sobre Information Literacy aumentou constantemente nesse período de dez anos. Os resultados revelaram também que a maioria das publicações de Information literacy foi escrita por autores da América e do Reino Unido.

Do mesmo modo, a pesquisa de Bhardwaj (2017) realizada com o objetivo de fazer uma análise quantitativa da literatura publicada sobre competência da informação em Ciências Sociais e Humanas no período 2001-2012, usando artigos indexados no banco de dados de citações do Scopus que cobre 67\% de publicações dos EUA, Canadá, Reino Unido, Holanda (Elsevier), Alemanha (Springer) e Suíça (Pipp, 2006) e com um menor número de publicações, os periódicos publicados na Ásia e na África demonstrou que a pesquisa sobre Competência em Informação (Colnfo) cresceu exponencialmente.

Estudos brasileiros recentes como a pesquisa de Leite et. al. (2016) em levantamento realizado no portal da ANCIB - ENANCIB, mostra que a partir de 2009 há um crescimento na produção de trabalhos sobre Colnfo apresentados no ENANCIB. Esse crescimento, além de expressivo também se dilui em diferentes grupos de pesquisa e com distintas aplicações, seja no contexto escolar e institucional, seja no ambiente corporativo.

Ampliando e, de certa forma ratificando o resultado anterior, a pesquisa de Belluzzo (2018) sobre a situação da CoInfo no contexto brasileiro definiu categorias de indicadores de produção científica da área Colnfo entre 2000 e 2016 e ao analisar a produção científica por tipo de produção e indicadores de assuntos, constatou que houve um desenvolvimento na produção cientifica de artigos de periódicos, teses e dissertações e livros.

O fato é o que o tema além de estar consolidado em muitos países no exterior na visão de Leite et al. (2016) a área vem despertando interesse no Brasil e vem crescendo no seu 
processo de institucionalização especialmente com o aumento da pesquisa e da pósgraduação nesse campo científico.

Em um quadro de consolidação da área em países desenvolvidos e de estágios iniciais e de expansão em países em desenvolvimento, importante se faz chamar atenção para a importância das parcerias e da criação e fortalecimento de redes nesse processo de construção do campo científico da Colnfo.

\section{0 papel das parcerias e redes no desenvolvimento das áreas científicas}

As parcerias são vistas como um esforço contínuo de cooperação para colaborar na geração de ideias visando um conjunto de propósitos mutuamente aceitáveis, e possibilitam expandir a atuação nas áreas de pesquisa, agregando mais valor ao trabalho (FORREST, 1992; HEMMINGS,1984; SHIVE E ROGUS,1979 citados por KISNER, MAZZA, LIGGETT,1997).

Consideradas como oportunidades na maior parte das vezes voluntárias, como troca de experiências e boas práticas, essas alianças surgem em interações com outras pessoas e especializações envolvidas e contribuem com uma ampla variedade de recursos baseados em objetivos compatíveis em relacionamentos contínuos e próximos, contribuindo para aumentar a credibilidade de uma organização, sua visibilidade e fornecendo uma rede em expansão para oportunidades dentro da organização.

A literatura destaca que para uma parceria ser considerada bem sucedida, devem existir compromissos mútuos que apresentam resultados de maior qualidade por meio de auxílios pontuais ou contínuos (HEMMINGS,1984; SHIVE E ROGUS,1979 citados por KISNER, MAZZA, LIGGETT,1997; STENGER-RAMSEY et. al., 2009).

Os parceiros devem ser escolhidos pelos pontos fortes que trazem para 0 relacionamento cujo objetivo é desenvolver sinergismo entre as contribuições dos parceiros, resultando em uma situação "win-win" para ambos ou todos eles (WALTERS, PETERS e DESS, 1994).

Assim, uma parceria proveitosa e transformacional consolida-se a partir do momento em que há o comprometimento por parte de todos os envolvidos em relação aos objetivos almejados conjuntamente (WALTERS, PETERS e DESS, 1994, citados por KISNER, MAZZA, LIGGETT , 1997).

Todas as partes devem ter missões compatíveis ou fins organizacionais, comprometerem-se com a parceria e compartilharem recursos livremente. Os envolvidos na gestão da parceria devem, em primeiro lugar, desenvolver uma missão conjunta, criar metas e objetivos claramente definidos. Devem manter uma comunicação aberta e regular para discutir tanto os resultados imprevistos e trabalhar para remover qualquer uma das seguintes 
barreiras à produtividade como a falta de confiança, a falta de continuidade, distribuição desigual de benefícios e custos, egos e o medo da mudança (STENGER-RAMSEY et. al., 2009).

Foi a partir de uma visão integrada sobre o papel das parcerias dessa ação conjunta a qual nos referimos nesse trabalho, iniciada com a identificação de objetivos compartilhados, que o grupo construiu mais concretamente os elementos que devem formar a base da parceria: objetivos comuns, compromissos individuais e organizacionais, liderança, colaboração, comunicação eficaz, tomada de decisão democrática, confiança e responsabilidade e que se acham descritos no Quadro1.

Quadro 1 - Desafios para uma Rede

\begin{tabular}{|c|c|c|c|}
\hline \multirow[b]{2}{*}{$\begin{array}{c}\text { Barreiras (1-6) e } \\
\text { compromissos para } \\
\text { parcerias em Redes }\end{array}$} & & Problemas & Soluções \\
\hline & $\begin{array}{l}1 . \\
2 . \\
3 . \\
4 .\end{array}$ & $\begin{array}{l}\text { Falta de confiança } \\
\text { Falta de continuidade } \\
\text { Falta de recursos } \\
\text { Distribuição desigual } \\
\text { de benefícios } \\
\text { Distribuição desigual } \\
\text { de custos } \\
\text { Egos e vaidades } \\
\text { Medo de mudanças }\end{array}$ & $\begin{array}{l}\text { - Objetivos comuns para as equipes } \\
\text { (integração de projetos) } \\
\text { - Compromissos e tarefas } \\
\text { individuais em sinergia } \\
\text { - Compromissos organizacionais } \\
\text { com agendas permanentes } \\
\text { - Colaboração e comunicação fluida } \\
\text { - Tomada de decisão democrática e } \\
\text { colaborativa, } \\
\text { - Confiança e responsabilidade na } \\
\text { rede. } \\
\text { - Humildade com os resultados }\end{array}$ \\
\hline
\end{tabular}

Fonte: Adaptado de STENGER-RAMSEY et. al. (2009).

Definiu-se a princípio, os principais elementos dessa ação conjunta, voltada ao fortalecimento da Colnfo na Ciência da Informação:

a) Integração do projeto/parceria em atividades de desenvolvimento institucional em todos os níveis;

b) Identificação dos parceiros em instituições brasileiras da área científica da área da Colnfo como universidades e institutos de pesquisa e agências - trabalho iniciado com a Universidade Estadual de São Paulo (Unesp), Universidade de Brasília (UnB), Universidade Federal da Paraíba, Instituto Brasileiro de Informação em Ciência e Tecnologia (IBICT) e a Associação Nacional de Pesquisa e Pós-graduação em Ciência da Informação (ANCIB);

c) Identificação no âmbito do IBICT de parceiros como a Rede Cariniana;

d) Definição com os participantes do projeto de cooperação científica, de propósitos específicos de cada parceiro, estratégias e compromissos individuais e institucionais destinadas ao desenvolvimento da ação que perpassa por incentivar a produção e 
divulgação de conhecimentos para a institucionalização desse campo científico na sociedade como um todo.

e) A adoção de níveis de colaboração, comunicação, tomada de decisão democrática e respeito às lideranças de cada parceria.

f) Acima de tudo cultiva-se a responsabilidade, respeito, generosidade e confiança entre pares.

\section{Instituições brasileiras integrando redes para o desenvolvimento da COINFO}

O Instituto Brasileiro de Informação em Ciência e Tecnologia (IBICT), reconhecendo a importância, cada vez maior, da informação científica em rede digital e do papel do IBICT como órgão de estabelecimento de políticas, coordenação de serviços de informação em C\&T\&l, desenvolvimento do ensino e da pesquisa em Ciência da Informação juntamente com a Unb, Unesp e ANCIB vêm atuando no fortalecimento da área de Competência em Informação (Colnfo). Para tanto têm realizado pesquisas e documentos técnicos sobre a Colnfo, planejado e participado, no Brasil, dos Seminários de Competência em Informação: Integrando as redes dos pesquisadores, ocorridos nos anos de 2014, 2015, 2016, 2017 e 2018 cujo o objetivo tem sido a criação de um espaço de reflexão, discussão e compartilhamento de experiências e práticas no campo disciplinar de Competência em Informação.

Vale ressaltar que o IBICT iniciou suas ações em Colnfo com ênfase na inclusão social e digital e nas estratégias de busca e uso da informação no ensino básico, com o Programa Corredor digital, já concluído, que capacitou professores e alunos de escolas rurais do distrito federal ensinando a lidarem com as novas tecnologias de informação aplicadas à educação. Em sequência às ações em prol do desenvolvimento da área "Competência em Informação"(Colnfo), o instituto em consonância com suas diretrizes anuais e atendendo ao compromisso assumido publicamente durante os seminários de Colnfo propôs o "Serviço de Informação e Comunicação Científica" em permanente interação com as instituições parceiras.

O objetivo do serviço, traduzido na criação de um repositório, uma biblioteca temática e ambiente virtual de pesquisa é desenvolver uma ambiência virtual de Informação e Comunicação na área de Competência em Informação para disponibilizar nesse campo temático, por meio de acesso livre, a produção científica dos pesquisadores da CoInfo no Brasil com a coleção de publicações digitais nacional e internacional em uma biblioteca virtual e um ambiente virtual de pesquisa. Dessa forma é possível fortalecer a colaboração dos pesquisadores nas atividades de ensino e pesquisa com vistas a institucionalização e desenvolvimento desse campo científico. Entre as principais características desses serviços pode-se destacar: 
a) O Repositório de Colnfo, um ambiente virtual que abrigará a produção científica digital produzida pelos pesquisadores da Colnfo com funcionalidades de submissão e depósito realizado por uma rede pesquisadores brasileiros da área da Colnfo;

b) A Biblioteca Virtual, um ambiente virtual que agregará documentos digitais a partir do desenvolvimento de uma coleção virtual nacional e internacional sobre tema que será alimentada por uma equipe contratada para tal finalidade;

c) Ambiente Virtual Colaborativo de Pesquisa - que reunirá funcionalidades de sala virtual de discussão, quadro de anotações, redação e abrigo de documentos oriundos dos eventos e deverá ter um pesquisador dessa temática para coordenar e animar a rede;

d) Agenda para a consolidação dos eventos e projetos.

O trabalho da equipe está alinhado ao conceito de acesso aberto que preconiza o acesso gratuito e irrestrito à produção técnica e científica digital, à preservação digital e respeito aos direitos autorais. Centra-se no oferecimento de Serviços de Informação e Comunicação Virtual voltados prioritariamente para pesquisadores, professores e estudantes, da área de Competência em Informação no Brasil. A proposta do referido Serviço de Informação e Comunicação foi discutida com os parceiros do projeto de Competência em Informação e aprovada pela comunidade científica da área de Coinfo em final de 2016 . No decorrer do ano de 2017, foi iniciada a elaboração do Repositório tendo sido identificada o DataVerse como plataforma para abrigar o conteúdo do Repositório.

O Data Verse é um repositório aberto de dados de todas as disciplinas, criado pelo Instituto de Ciências Sociais Quantitativas da Universidade de Harvard para que os pesquisadores possam compartilhar e manter o controle de seus documentos e dados e da qual faz parte a Rede Brasileira de Serviços de Preservação Digital do IBICT (Rede Cariniana), com o objetivo de garantir o acesso contínuo a documentos a longo prazo. Essa ferramenta foi originalmente criada para receber dados de pesquisa, mas tem funcionalidades que permitem o depósito e recuperação de publicações científicas (DATA VERSE, 2018; INSTITUTO, 2012; 2018).

Assim, em ação conjunta com a Coordenação da Rede Cariniana, responsável pela rede de preservação do IBICT, foi instalada pela equipe da Cariniana a plataforma DataVerse da Colnfo e, em seguida, foi elaborado em ação conjunta com a equipe da Cariniana e do Projeto Brasília 2060 o "Guia de orientação para submissão dos trabalhos no Dataverse Colnfo", tendo sido definido também o conjunto de metadados para o repositório. 
O referido Guia foi debatido no Enancib de 2016 e enviado para pesquisadores da Colnfo, por ocasião do pré-teste realizado no mês de junho de 2017 para verificar as facilidades e ou dificuldades de usos da ferramenta pelos usuários. Dos seis pesquisadores contatados para realizarem o pré-teste, três alimentaram a plataforma sem maiores problemas. Essa sondagem inicial permitiu que a equipe fizesse ajustes e debatesse sobre a questão no seminário de Colnfo, realizado desta vez em Marília (SP), durante a programação do Pós-Enancib de 2017.

Em 2018, foi elaborado, em ação conjunta com a Rede Cariniana e o Projeto Brasília 2060, o documento "Proposta de diretrizes para desenvolvimento de políticas em repositórios de documentos científicos" que servirá para o desenvolvimento das políticas do Repositório da Colnfo, já apresentado para análise no evento da ANCIB de 2018.

Em seguida, optou-se por criar a Biblioteca Virtual da Coinfo utilizando para abrigar sua coleção, também a plataforma Data Verse, cujas especificações foram mencionadas. O documento de planejamento e metadados já foram definidos e a equipe agora trabalha no manual para desenvolvimento e alimentação da coleção da BV Colnfo. Foi também planejada a interface que integrará os dois serviços de informação: repositório e biblioteca virtual. A submissão de documentos, busca e recuperação da informação é realizada na ambiência do DataVerse.

Essa ação de reunir e disponibilizar conteúdos de informação e oferecer serviços de informação e comunicação está em sintonia com o pensamento de Ziman (1984) citado por Leite et al. (2016) que considera que o acesso à literatura primária se dá especialmente por meio dos periódicos científicos e por sistemas de recuperação dessa informação científica.

Ainda, nessa mesma linha de pensamento voltada à ênfase para os serviços de informação em ciência e tecnologia, Garvey e Griffith (1979) citados por Leite et al. (2016) afirmam que a comunicação científica incorpora atividades associadas à produção, disseminação e uso da informação desde o momento em que o cientista concebe uma ideia para pesquisar até que a informação acerca dos resultados seja aceita como estoque universal de conhecimentos.

Outro argumento para uma ação de Serviços de Informação na Colnfo refere-se ao nexo que a área da CoInfo tem com a aprendizagem e o ciclo da informação. Essa ideia apoiase no que Belluzzo (2005) afirma sobre a aprendizagem, quando ressalta que ela passa por um processo de organização das informações e de sua integração à estrutura cognitiva. Enfatiza a autora que essa aprendizagem somente será significativa quando a nova informação for assimilada ao conhecimento de quem aprende. 
A ideia do serviço de informação como instrumento de fortalecimento da rede de Colnfo é defendida na utilização dos princípios da competência em informação em sintonia com os serviços especializados da classe bibliotecária. Nesse cenário tem importância a Rede de Bibliotecas das Unidades de Pesquisa do Ministério da Ciência, Tecnologia e Inovação (RBP/MCTI), que foi criada em 2009, sendo o seu principal objetivo promover o compartilhamento e cooperação entre os institutos de pesquisa do MCTIC para intensificar o acesso à informação da pesquisa científica e tecnológica.

Uma aliança estratégica foi estabelecida entre o IBICT/RPB e a UnB, criando-se o “Projeto Integrador entre as Bibliotecas de Pesquisa e as Bibliotecas Universitárias: Competência em Informação, Bibliotecas Universitárias e Bibliotecas de Pesquisa", voltado a uma ação integrada na formação em Ciência e Tecnologia a partir de organização de uma rede colaborativa com instituições de ensino e de pesquisa para prover conhecimento de práticas de pesquisa e uma didática para a produção de conhecimento em C \& T e ampliar o modelo de qualificação profissional promovendo o diálogo entre bibliotecários nas ações cooperativas e multidisciplinares, além de estimular a pesquisa em Competência em Informação (SEMINÁRIO Colnfo, Pós-Enancib, 2016).

Em pesquisa realizada como parte desse projeto os autores Santos, Simeão, Belluzzo (2014) procuram mostrar como a interação das Bibliotecas Universitárias com as Bibliotecas de Unidades de Pesquisa do MCTIC (RBP), por exemplo, é um movimento importante para a consolidação das parcerias e ampliação das áreas envolvidas, isto porque pode propiciar colaboração na formação integral dos alunos e estimular profissionais da informação a produzirem conhecimento, formar e desenvolver coleções adequadas para cada área, bem como promover o acesso ao conhecimento e a informação observando com as particularidades de cada área, além da necessidade de se criar um conjunto de indicadores quantitativos e qualitativos que permitam avaliar a aprendizagem digital e informacional em ambientes tecnológicos, oferecendo informações importantes para os diretores e coordenadores das unidades acadêmicas e demais professores. É fundamental segundo os autores, compartilhar experiências e projetar as estratégias em informação científica e tecnológica realizadas por esse grupo de especialistas, notadamente no ambiente universitário e de pesquisa.

Essa proposta/ação do IBICT/RBP tem sido discutida e referendada pelos parceiros e encontra eco na tendência de uma nova modelagem para grupos de pesquisas, publicações em pares, treinamento de pesquisa, socialização e cooperação. É um pensamento que se alinha ao que defendem vários estudiosos, dentre eles, Rodríguez, Bertone, García-Martínez (2010), entre outros. Desse modo, o projeto de parceria entre Bibliotecas Universitárias com as 
Bibliotecas de Unidades de Pesquisa do MCTIC (RBP) tem como propósitos de cooperação e compartilhamento para:

a) Fomentar a qualificação científica do profissional da informação (híbridos) promovendo o diálogo com pesquisadores nas ações cooperativas e multidisciplinares para a produção de conhecimento.

b) Contribuir para a cultura do reuso de dados e da formação do bibliotecário de dados.

c) Conceber um programa de disseminação do conhecimento científico e tecnológico a partir do apoio aos Programas de Formação nas Bibliotecas participantes.

d) Contribuir para a melhoria dos processos de recuperação e uso da informação, ajudando na formação integral nas universidades, preparando os alunos (graduação e pós-graduação) para os desafios do mercado de trabalho, com excelência e inovação.

e) Divulgar as ações das unidades de pesquisa no âmbito das universidades. (SEMINÁRIO Colnfo, Pós-Enancib, 2016).

Esse projeto apoiou-se na metodologia que se apresenta na Figura 1.

Figura 1 - Metodologia desenvolvida no projeto

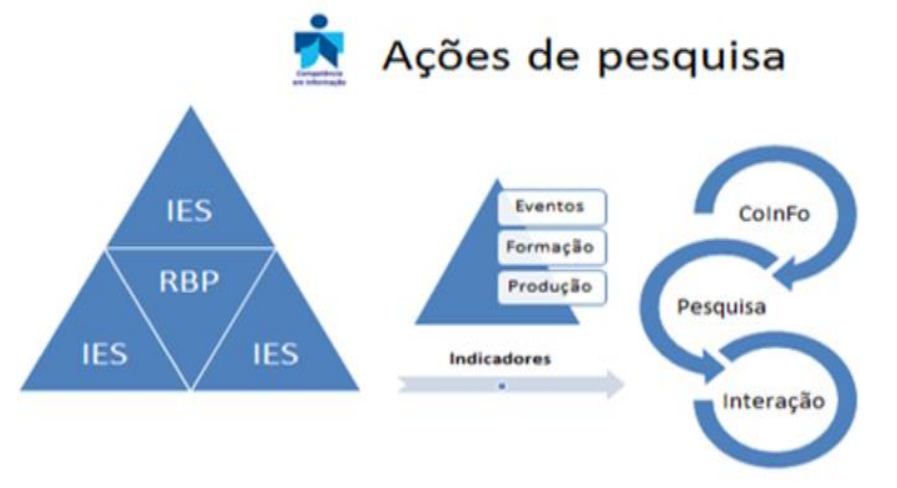

Fonte: as autoras.

Como decorrência de uma parceria entre o IBICT/RBP/ UnB e em articulação com o "Projeto Integrador entre as Bibliotecas de Pesquisa e as Bibliotecas Universitárias: Competência em Informação, Bibliotecas Universitárias e Bibliotecas de Pesquisa", foi criado, achando-se em desenvolvimento, um novo projeto com a participação da Universidade Estadual Paulista (UNESP) intitulado "Promoção de rede colaborativa e uso estratégico de recursos para formação continuada: desenvolvimento da competência em informação com foco no perfil pesquisador dos bibliotecários da biblioteca da FFC/UNESP - campus de Marília", enquanto um piloto para a sua aplicação, futuramente, a toda a Rede de Bibliotecas da UNESP, esperando que possa servir como norteador ao alcance de resultados, tais como: 
- Adotar um conceito Colnfo levando em consideração as características da Instituição;

- Fazer com que os bibliotecários tenham claros os elementos que compõem a Sociedade da Informação, do Conhecimento, da Aprendizagem, do novo paradigma informacional e tecnológico e sua dinâmica, em inter-relação com os princípios da Colnfo;

- Identificar e inserir elementos da competência em informação na missão, visão, valores, objetivos e metas da Biblioteca; e,

- Verificar a competência em informação dos bibliotecários da Instituição, a partir do uso de Padrões sobre Colnfo, focando em seu perfil pesquisador (SANTOS, C., 2017).

A concepção do projeto acha-se articulada ao conceito de que é preciso que os Conhecimentos, as Habilidades e as Atitudes (CHA) dos bibliotecários universitários competentes em informação devam ser aplicados na realização e na promoção das pesquisas científicas (SANTOS, R., 2017, p. 172) e na Estrutura de Framework - Programa de Colnfo (Adaptado de SANTOS, C., 2017) (Figura 2), criando-se um programa de natureza modular para o seu desenvolvimento.

Figura 2 -Framework para Colnfo

\begin{tabular}{|c|c|c|c|c|}
\hline Ideia Central & Marcos Gerais & Linhas de Ação & $\begin{array}{c}\text { Conteúdos } \\
\text { Abordados }\end{array}$ & Cronograma \\
\hline $\begin{array}{c}\text { Contextualização } \\
\text { de cenários e de } \\
\text { conceitos }\end{array}$ & $\begin{array}{c}\text { Conjunto de } \\
\text { disposiçães } \\
\text { didáticas para a } \\
\text { operacionalização } \\
\text { da ideia central }\end{array}$ & $\begin{array}{c}\text { Aplicação dos } \\
\text { marcos gerais }\end{array}$ & $\begin{array}{c}\text { Temas para } \\
\text { discussões }\end{array}$ & $\begin{array}{c}\text { Período das } \\
\text { atividades }\end{array}$ \\
\hline
\end{tabular}

Fonte: Santos, C. (2017).

Na prática, essa concepção de fazer pesquisa em rede traz transformações ao universo de instituições que trabalham com o ciclo informacional, refletindo-se na criação de unidades de informação de ambientes acadêmicos mais colaborativos. Segundo Blattmann, Weber (2008) também tem o objetivo de reunir, preservar e disseminar a produção científica com vistas ao acesso e uso de seus acervos de forma eletrônica/digital na internet, compartilhando recursos informacionais.

\section{Considerações finais}

Considera-se que "Redes", "estratégia coletiva", "empreendimentos conjuntos", "alianças estratégicas" integram a administração estratégica fortalecendo os relacionamentos cooperativos e a formação de estratégias para tornar-se um processo conjunto a ser 
desenvolvido com parceiros (MINTZBERG, AHLSTRAND, LAMPEL, 2000), o que é um requisito de sucessos às ações de pesquisa em ciência e tecnologia no contexto brasileiro.

O estabelecimento de parcerias e alianças estratégicas por organizações que oferecem serviços se torna cada vez mais relevante visto que os serviços têm características especiais em suas operações conforme Gianesi e Corrêa (2007, p. 32) como "a intangibilidade dos serviços, a necessidade da presença do cliente e o fato de que geralmente os serviços são produzidos e consumidos simultaneamente", o que tem importância também para as bibliotecas e unidades de serviço de informação.

Por meio de parcerias e de alianças estratégicas entre instituições brasileiras, embora ainda em situação emergente, foi possível criar e desenvolver projetos como parte de vivências que foram apresentadas com foco na consolidação da Colnfo como uma área da Ciência da Informação, abrir frentes de atuação, ampliar o leque de conhecimentos, captar e otimizar recursos e aumentar a capacidade de intervenção na realidade entre as bibliotecas de institutos de pesquisa e as bibliotecas universitárias.

\section{Referências}

BELLUZZO, Regina Célia Baptista. Competência em informação: um diferencial das pessoas no século XXI. [slide]. Campinas: Unicamp, 2005. Disponível em: https://www.fe.unicamp.br/eventos/getic/arquivos/Oficina Regina.pdf Acesso em: 10 jul. 2018.

BELLUZZO, Regina Célia Baptista. A competência em informação no Brasil: cenários e espectros. São Paulo: ABECIN, 2018. Disponível em: http://abecin.org.br/data/documents/EBook Belluzzo.pdf Acesso em: 27 jun. 2018.

BHARDWAJ, Raj Kumar. Information literacy literature in the social sciences and humanities: a bibliometric study. Information and Learning Science, v. 118, n. 1-2, 2017, p. 67-89, 2017. Disponível em: https://doi.org/10.1108/ILS-09-2016-0068 Acesso em: 10 jul. 2018.

BRASIL. MINISTÉRIO DE CIÊNCIA E TECNOLOGIA. Relatório geral do evento: seminário sobre competência em informação do ENANCIB: integrando as redes dos pesquisadores: proposta..., 1, Belo Horizonte, 2014. Brasília, DF: [s.n.], 2014. Disponível em: http://www.Iti.pro.br/userfiles/downloads/RELATORIO seminario ENANCIB 2014.pdf Acesso em: 29 jul. 2018.

DATAVERSE PROJECT, 2018. Disponível em: https://dataverse.org/ Acesso em: 22 set. 2018.

DUDZIAK, Elisabeth Adriana. Competência informacional: análise evolucionária das tendências da pesquisa e produtividade científica em âmbito mundial. Informação \& Informação, Londrina, v. 15 , n. 2, p. 1-22, 2010. Disponível em: http://dx.doi.org/10.5433/19818920.2010v15n2p1 Acesso em: 19 ago. 2018.

GIANESI, Irineu Gustavo Nogueira.; CORREA, Henrique Luís. Administração estratégica de serviços: operações para a satisfação do cliente. São Paulo: Atlas, 2007.

INSTITUTO BRASILEIRO DE INFORMAÇÃO EM CIÊNCIA E TECNOLOGIA (IBICT). Rede Brasileira de Serviços de Preservação Digital: Cariniana. 2012. Disponível em: 
http://cariniana.ibict.br/index.php/publicacoes/item/27-politica-da-rede-brasileira-deservicos-de-preservacao-digital Acessado em: 22 set. 2018.

INSTITUTO BRASILEIRO DE INFORMAÇÃO EM CIÊNCIA E TECNOLOGIA (IBICT). Dataverse do IBICT. 2018. Disponível em: https://repositoriopesquisas.ibict.br Acessado em: 25 jun. 2018.

KISNER, Mary J, MAZZA Maralyn J.; LIGGETT, David R. Building Partnerships. New Directions for Community Colleges, n. 97, Spring 1997, p. 23-28. Disponível em: https://onlinelibrary.wiley.com/doi/epdf/10.1002/cc.9703 Acesso em: 15 set. 2018.

LEITE, Cecília et al. Cenário e perspectiva da produção científica sobre competência em informação (Colnfo) no Brasil: estudo da produção no âmbito da ANCIB. Informação \& Sociedade: Estudos, João Pessoa, v. 26, n. 3, p. 151-168, set./dez. 2016. Disponível em: http://www.periodicos.ufpb.br/ojs2/index.php/ies/article/view/31983/16876 Acesso em: 25 jun. 2018.

MAJID, SHAHEEN et al. Analyzing publishing trends in information literacy literature: a bibliometric study. Malaysian Journal of Library \& Information Science, v. 20, n. 2, p. 51-66, 2015. Disponível em: https://ejournal.um.edu.my/index.php/MJLIS/article/view/1766 Acessado em: 25 jun. 2018.

MINTZBERG, Henry; AHLSTRAND, Bruce; LAMPEL, Joseph. Todas as partes do elefante. HSM management, n. 12, p. 100-108, jan./fev. 1999.

RODRÍGUEZ, Dario; BERTONE, Rodrigo; GARCÍA-MARTÍNEZ, Ramón. Collaborative research training base on virtual spaces. In: REYNOLDS, N., TURCSÁNYI-SZABÓ, M. (Eds.) Key competences in the knowledge society: IFIP/TC International Conference. Berlin: SpringerVerlag, 2010. p. 344-353.

SANTOS, Rafael Barcelos, SIMEÃO, Elmira Luzia Melo Soares; BELLUZZO, Regina Célia Baptista, 2014. Competência em Informação (coinfo) no bibliotecário protagonista: estudo do perfil da rede de bibliotecas de Pesquisa do MCTIC à luz do Diagrama Belluzzo. Inclusão Social, Brasília, v. 8, n.1, p. 89-100, jul./dez. 2014. Disponível em: http://revista.ibict.br/inclusao/article/view/3025/2767 Acesso em: 29 jun. 2018.

SANTOS, Camila Araújo dos, 2017. Competência em informação na formação básica dos estudantes da educação profissional e tecnológica [online]. Tese de doutorado, Faculdade de Filosofia e Ciências, Universidade Estadual Paulista Júlio de Mesquita Filho, 2017. Disponível em: https://repositorio.unesp.br/handle/11449/150036 Acesso em: 29 out. 2018.

SEMINÁRIO SOBRE A COMPETÊNCIA EM INFORMAÇÃO, 2015. Pós-Enancib, João Pessoa. Relatório. Pesquisa Brasileira em Ciência da Informação e Biblioteconomia, João Pessoa, v. 10, n. 2, p. 379-383, 2015.

SEMINÁRIO SOBRE A COMPETÊNCIA EM INFORMAÇÃO, 2016. Pós-Enancib, 2016. Relatório final do III seminário sobre competência em informação do Enancib. Salvador, 2016.

STENGER-RAMSEY et. al. Building a challenge course? The important role of partnerships. In: ASSOCIATION OF OUTDOOR RECREATION \& EDUCATION CONFERENCE. Proceedings. 2009, p. 69-75.

WALTERS, Bruce A., PETERS, Steve; DESS, Gregory G. Strategic alliances and joint ventures: making them work. Business Horizons, v. 37, n. 4, p. 5-10, July/August 1994. Disponível em: https://www.sciencedirect.com/science/article/pii/0007681394900418 Acesso em: 26 jun. 2018. 